\section{DIGNIFICANDO LA GESTIÓN DE RIESGO DE DESASTRES: LIDERAZGOS FEMENINOS Y ESTRATEGIAS COMUNITARIAS EN EL CAMPAMENTO DIGNIDAD, SANTIAGO DE CHILE}

Valentina Acuña ${ }^{1^{*}}$, Sofía Valdivieso ${ }^{1}$ y Leila Juzam ${ }^{1}$

\section{RESUMEN}

Recientemente, se ha problematizado cómo la Gestión de Riesgo de Desastres, o GRD, conceptualiza la resiliencia y los desastres en asentamiento informales. Mediante un estudio de caso cualitativo y una aproximación que problematiza la escala local, mostramos qué es considerado amenaza por la comunidad y de qué manera se gestiona. De esta manera evidenciamos que las comunidades se enfrentan a múltiples amenazas -pandemia, riesgo de aluvión, inseguridad alimentaria, escasez hídrica, inundaciones e incendios- que son gestionadas partir de estrategias que descansan en la organización comunitaria y redes de solidaridad externas e internas al territorio bajo liderazgos femeninos, colaboración equitativa, apoyo voluntario, identificación política y transmisión de conocimientos locales. Nuestros resultados sugieren dos grandes consideraciones a la GRD en asentamientos informales. Primero, que el concepto de resiliencia en tanto adaptación, debe expandirse a partir de voces subalternas y la noción de dignidad como categoría moral para la transformación, dejando de lado ideas de estabilidad y despolitización. Y segundo, que esta debe estimular políticas de desarrollo que se hagan cargo del problema habitacional y desigualdades de género, problematizando las formas particulares de relación y organización, y por ende, las categorías tradicionales de hogar.

\section{PALABRAS CLAVES}

Asentamientos informales, Resiliencia, Múltiples amenazas, Dignidad, Gestión comunitaria del riesgo, Gestión de riesgo de desastres

\section{DIGNIFYING DISASTER RISK MANAGEMENT: FEMALE LEADERSHIP AND COMMUNITY STRATEGIES IN CAMPAMENTO DIGNIDAD, SANTIAGO DE CHILE}

\section{ABSTRACT}

Recently, it has been problematized how Disaster Risk Management, or just DRM, conceptualizes resilience and disasters in informal settlements. Through a qualitative case study and an approach that problematizes the local scale, we show what is considered a hazard by the community and how it is managed. In this way we show that communities face multiple hazards - pandemic, landslide risk, food insecurity, water shortage, floods, and fires - that are managed from strategies that rely on community organization and external and internal solidarity networks to the territory under female leadership, equitable collaboration, voluntary support, political identification and transmission of local knowledge. Our results suggest two major DRM considerations in informal settlements. First that the concept of resilience as adaptation must expand from subaltern voices and the notion of dignity as a moral category for transformation, leaving aside ideas of stability and depoliticization. And second, that it should stimulate development policies that take into account the housing problem and gender inequalities, problematizing the particular forms of relationship and organization, and therefore, the traditional categories of home.

\author{
1. Centro de Investigación \\ para la Gestión Integral \\ del Riesgo de Desastres \\ CIGIDEN, Santiago, Chile. \\ *Autor de correspondencia: \\ vmacuna@uc.cl
}

Identificador: http://revistareder.com/ handle-0719-8477-2021-096

\section{RECIBIDO}

10 de febrero de 2021

\section{ACEPTADO}

22 de marzo de 2021

PUBLICADO

1 de julio de 2021

\section{Formato cita}

Recomendada (APA): Acuña, V., Valdivieso,

S. \& Juzam, L. (2021). Dignificando la Gestión de Riesgo de Desastres: liderazgos femeninos y estrategias comunitarias en el Campamento Dignidad, Santiago de Chile. Revista de Estudios Latinoamericanos sobre Reducción del Riesgo de Desastres REDER, 5(2), 91106. http://revistareder.com/ handle-0719-8477-2021-096

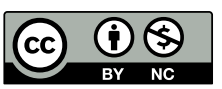

Todos los artículos publicados en REDER siguen una política de Acceso Abierto y se respaldan en una Licencia CreativeCommons Atribución-NoComercial 4.0 Internacional.

\section{Revista de Estudios} Latinoamericanos sobre Reducción del Riesgo de Desastres (REDER)

Diseño: Lupe Bezzina

\title{
KEYWORDS
}

Informal settlements, Resilience, Multiple hazards, Dignity, Communitarian risk management, Disaster risk management 


\section{INTRODUCCIÓN}

Los asentamientos informales "se desarrollan fuera de los sistemas legales que buscan reconocer la tenencia y posesión de la tierra e imponer el cumplimiento de las regulaciones que se relacionan con el uso y planificación de la tierra, estructuras construidas, y seguridad y salud pública" (Satterthwaite et al., 2018, p.143). Más concretamente, son "áreas donde grupos de unidades de viviendas han sido construidas en tierras sobre las que los ocupantes no tienen derecho legal o que ocupan ilegalmente" (UN, 1997). El Estado de Chile los define como "campamentos" por corresponder a una ocupación precaria e irregular de terrenos con más de 8 hogares, que carecen de uno o más servicios básicos ${ }^{1}$ (MINVU, 2019).

El pasado 25 de marzo de 2021 la ONG latinoamericana $\mathrm{TECHO}^{2}$ publicó cifras alarmantes respecto a la situación actual de este tipo de asentamientos en Chile. Al día de hoy, 81.643 familias viven en 969 campamentos siendo la cifra histórica más alta desde 1996 (TECHO, 2021). Esta situación se recrudece si se considera la alta correlación existente entre el grado de afectación de desastres y los grupos sociales que habitan en zonas de exposición (UN-Habitat, 2003; Ward et al., 2016; Sandoval \& Sarmiento, 2020). Esto último es patente en distintas regiones de Chile, por ejemplo en Antofagasta los habitantes de campamentos están expuestos a amenazas por remoción en masa, y en los cerros de Valparaíso a incendios forestales. La pandemia, por su parte, también tiene un grado de afectación en asentamientos informales, ya que esta exacerba las inequidades estructurales de los territorios debido las altas densidades residenciales, carencias de acceso a salud, ocupaciones de alto riesgo y la incapacidad de llevar a cabo las estrategias gubernamentales de aislamiento, distanciamiento social y cuarentena (Mitlin, 2020). En efecto, desde América Latina se ha relevado que la pandemia es sólo una condición más de riesgo en sectores populares (Allen et al., 2020) y que su influencia exacerba la acumulación de riesgos previos (González et al., 2020; Allen, 2018).

A pesar de esta evidente correlación, tanto en América latina como en Chile, los asentamientos informales suelen estar excluidos de los procesos formales de gobernanza urbana (Herrle, Ley \& Fokdal, 2015; Sandoval \& Sarmiento, 2020). En Chile en particular, esto es dramático ya que si bien, los avances recientes en Gestión de Riesgo de Desastres-GRD- han tenido el propósito de superar los enfoques reactivos, las familias que viven en asentamientos informales no han sido prioridad en las acciones de prevención, mitigación y preparación ante desastres (Flores et al., 2019). Por esta razón, se argumenta, que la marginación de las personas que viven en campamentos, es económica, social e institucional ya que usualmente, están fuera de las políticas públicas urbanas que están diseñadas para la población "formal" (Herrle, Ley \& Fokdal, 2015).

A nivel general, la GRD ha puesto el foco en la resiliencia con una tendencia a normativizar y posicionarla como una meta a desear (Cote \& Nightingale, 2012; Vale, 2014). No obstante, presta poca atención a las dimensiones de poder, escala, y equidad que le son inherentes (Cote \& Nightingale, 2012; Cretney, 2014; Evans, 2011; MacKinnon \& Derickson, 2012; Pizzo, 2015; Weichselgartner \& Kelman, 2015). Es por esto que desde las ciencias sociales se ha problematizado críticamente sus suposiciones subyacentes (Barrios, 2016), teniendo en cuenta ciertos elementos claves: dejar de pensar la resiliencia en función de la estabilidad y despolitización de los sistemas socioecológicos y construir definiciones comunes que prioricen voces subalternas y apunten a una transformación de los regímenes de riesgo (Solecki et al., 2017).

El Campamento Dignidad, ubicado en Quebrada de Macul, permite poner en discusión los elementos mencionados anteriormente. Este fue resultado de una ocupación ilegal de terreno originada en el contexto de la revuelta social en Chile durante octubre de 2019 , y ya a fines del año 2020 albergaba a más de 600 familias en la ribera norte de una de las quebradas más activas del piedemonte de Santiago, en medio de la pandemia por COVID-19. Como veremos a continuación, cuando se utiliza una mirada que problematiza la escala de lo local atendiendo a la experiencia cotidiana de las personas, se observa que las amenazas son múltiples y diarias (BullKamanga et al., 2003). A partir de un estudio de caso, vemos que la organización comunitaria y las estrategias empleadas para hacer frente a amenazas múltiples problematizan la conceptualización de resiliencia como capacidad adaptativa y su movilización al interior de los regímenes de gestión del riesgo en el territorio en particular, y en asentamientos informales a nivel nacional.

A modo de situar nuestro argumento, en la primera sección revisamos cómo se moviliza comúnmente el concepto de resiliencia en los estudios de desastres tomando en
1. Gas, luz, agua potable y concesiones sanitarias.

2. Fundación sin fines de lucro, cuyo propósito es combatir la exclusión construyendo ciudades justas y sostenibles (https://www.techo.org/ chile/que-es-techo). 
consideración las visiones críticas provenientes de las ciencias sociales a modo de extender el concepto de resiliencia como capacidad adaptativa hacia el de transformación. En la segunda sección, mostramos los resultados de la investigación realizada en el Campamento Dignidad dando cuenta de las características de la organización comunitaria y las estrategias para hacer frente a múltiples amenazas, y la importancia de los liderazgos femeninos en su constitución. Finalmente, concluimos en primer lugar, que el concepto de resiliencia debe construirse prestando atención a voces subalternas, dejando de lado ideas de estabilidad y despolitización a través de la consideración de la noción de dignidad como categoría moral. Y segundo lugar, que esta debe estimular políticas de desarrollo que se hagan cargo del problema habitacional y desigualdades de género, problematizando las formas particulares de relación y organización, y por ende, de las categorías tradicionales de hogar.

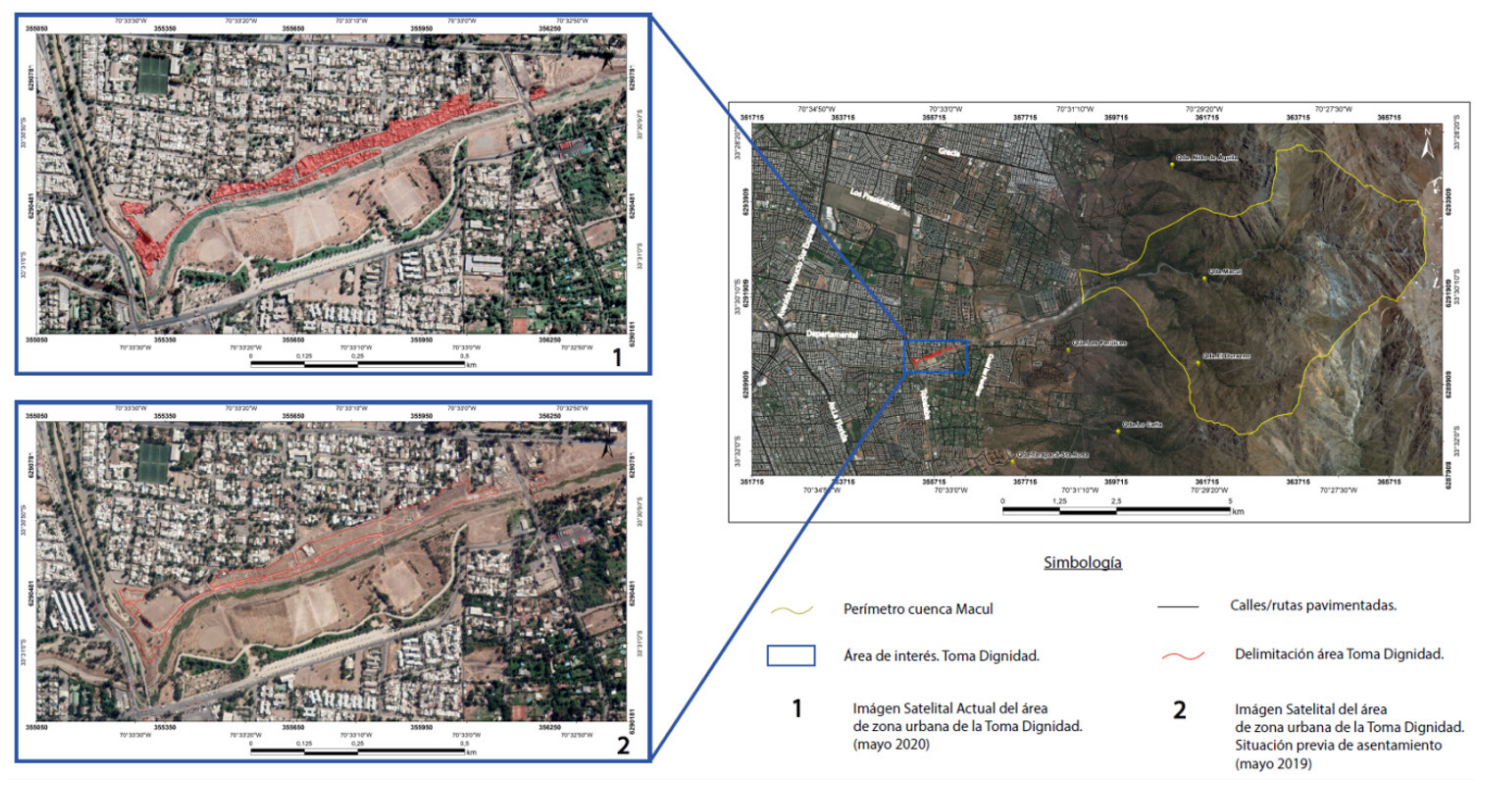

Figura 1. Área de zona urbana Campamento Dignidad mayo 2019 y 2020 Fuente: Francisca Roldán, 2020.

\section{HACIA UNA VISIÓN CRÍTICA DE LA RESILIENCIA}

El concepto de resiliencia, heredero de la ecología (Brown, 2014; Garschagen, 2013; Meerow \& Newell, 2015), fue seminal para la elaboración de las teorías de los sistemas socio-ecológicos donde se identifica como la cantidad de perturbación que puede sostener un sistema sin perder sus funciones clave o cambiar de estado, capacidad de auto-organización, adaptación y aprendizaje (Folke et al., 2002). Y por ello, se ha convertido en un elemento central para pensar cómo sistemas complejos pueden persistir de cara a la incertidumbre, disrupción y cambio (Davoudi et al., 2012; Maytas \& Pelling, 2014). Esta perspectiva, ha adquirido especial relevancia en el caso de los estudios urbanos donde se ha tendido a normativizar y posicionar como una meta a desear (Cote \& Nightingale, 2012; Vale, 2014).

No obstante lo anterior, las ciencias sociales, entre ellas la geografía, han sido críticas al concepto de resiliencia así entendido, argumentando que presta poca atención a las dimensiones de poder, escala, y equidad que le son inherentes (Cote \& Nitghtingale, 2012; Cretney, 2014; Evans, 2011; MacKinnon \& Derickson, 2012; Pizzo, 2015; Weichselgartner \& Kelman, 2015). Parte del problema, se encontraría en la transferencia poco crítica que se ha hecho de un concepto proveniente de la ecología a los sistemas sociales (Brown, 2014), y en cómo, quiénes y para qué se ha implementado (Elmqvist, 2014). Kaika (2017) enfatiza al respecto, que los llamados a "ciudades seguras, resilientes, sostenibles e inclusivas" es un camino que depende de viejas herramientas metodológicas, soluciones tecno-gerenciales y marcos institucionales propios del paradigma de modernización ecológica que "vacuna a los ciudadanos y al medio ambiente para que puedan soportar mayores dosis de desigualdad y degradación en el futuro; mediando los efectos de la desigualdad socio ambiental global, pero hace poco para aliviarla " (Kaika, 2017, p.89).

Al respecto, la antropología del desastre ofrece una explicación detallada de los aspectos problemáticos del concepto de resiliencia al examinar críticamente sus suposiciones subyacentes 
(Barrios, 2016). En primer lugar, el concepto de resiliencia asume la idea de estabilidad o retorno al estado anterior a la perturbación (estado de equilibrio). Lo problemático de esta suposición es que los desastres y comunidades humanas están lejos de ser estables (Brightman, 1995; Gupta \& Ferguson, 1992). En efecto, la antropología ha sido enfática en mostrar que la composición, estructura social y valores de las comunidades cambian como resultado de influencias externas e internas. Asimismo, los desastres crean condiciones en las cuales nacen comunidades emergentes (Fortun, 2001). Entonces, un concepto de resiliencia que asume la estabilidad de los sistemas sociales, no es suficiente para dar cuenta de los arreglos político-sociales que caracterizan a los desastres (Barrios, 2014; 2016).

En segundo lugar, la resiliencia así entendida corre el riesgo de despolitizar procesos que son profundamente políticos e históricos marcados por el colonialismo, la conformación de los EstadoNación y procesos de desarrollo (Hsu et al., 2015; Oliver-Smith, 1999). Más específicamente, la ecología política sugiere que los desastres han estado y están moldeados por prácticas humanas que aumentan las capacidades disruptivas de los fenómenos geofísicos, distribuyendo inequitativamente sus efectos en base a diferenciaciones de género, raza y clase. Los énfasis de los enfoques predominantes de resiliencia articulan así, narrativas despolitizadas que borran los procesos históricos mediante los cuales la vulnerabilidad ante desastre se produce, y donde la falta de resiliencia se reduce a una lógica victimizante. Así, el concepto de resiliencia como la capacidad de los sistemas para enfrentar o adaptarse a amenazas de desastres, más que mitigar, alimenta el mantenimiento del sistema en sí mismo.

Desde esta perspectiva, la resiliencia es una capacidad que va de la mano de la producción de la vulnerabilidad más que de su reducción. Por esta razón, el foco debería situarse en la construcción de definiciones de resiliencia que prioricen voces subalternas como mecanismos para abordar las prácticas de injusticia que rutinariamente dan forma a la vulnerabilidad ante desastres, y que permitan avanzar hacia transformaciones en el manejo del riesgo (Boano, 2017).

Solecki et al. (2017) caracteriza los regímenes de manejo del riesgo como el conjunto de instituciones, normas, y comportamientos organizados de tal manera que promueven un patrón arraigado de preparación, respuesta y recuperación frente al riesgo de desastres. Y llevan incrustados un conjunto de supuestos y afirmaciones sobre el riesgo, derechos y responsabilidades del estado, otros actores, el modo de gobernanza y los problemas subyacentes de equidad y justicia (Solecki et al., 2017). En efecto, en el área de estudio, el régimen de manejo del riesgo ha sido problematizado por Fuentealba y Verrest (2020), al conceptualizarlo como una gobernanza tecno-gerencial basada en la implementación de políticas de uso de suelo y construcción de infraestructuras de mitigación, como una estrategia importante de despolitización.

No obstante, a raíz de esta carencia, también se ha evidenciado la emergencia de iniciativas ciudadanas comunitarias frente al riesgo (Biskupovic, 2019), las que responden al vacío dejado por el paradigma dominante. El caso del Campamento Dignidad basado en una aproximación que prioriza la escala local entendida como el nivel en el que las amenazas se experimentan y afectan la vida cotidiana de las personas (Tironi et al., s/f) ${ }^{3}$, da cuenta de la necesidad de prestar atención a cómo desde la lucha por tener una vida digna (Pérez, 2018) emergen formas de acción colectivas que exigen dignificar una vida en un contexto de exposición a amenazas múltiples. Con el objetivo de relevar estas formas alternativas de gestionar los riesgos, argumentamos, se debe expandir el concepto de resiliencia en tanto capacidad adaptativa, apuntando hacia una transformación (Solecki et al., 2017) de la configuración del sistema de la mano de la construcción de nuevos principios morales para la GRD en asentamientos informales que incluya la categoría moral de dignidad (Pérez, 2018).

\section{APROXIMACIÓN METODOLÓGICA}

La metodología utilizada fue un estudio de caso cualitativo (Yin, 2015) de aproximación mixta (digital e in situ). De la misma forma en que los estudios de desastres plantean el valor de la capacidad de adaptación de los territorios y poblaciones luego de que ocurre un desastre, la comunidad científica ha tenido que adaptarse y buscar nuevas rutas y herramientas para cumplir sus objetivos y curiosidad -etnográfica en nuestro caso- en medio de uno de los más grandes desastres de los últimos años: la pandemia producto del COVID-19. Es por esto que las formas de recolección de datos cualitativos a partir de medios digitales se han vuelto una herramienta clave para los investigadores e investigadoras sociales desde que comenzó la emergencia sanitaria.

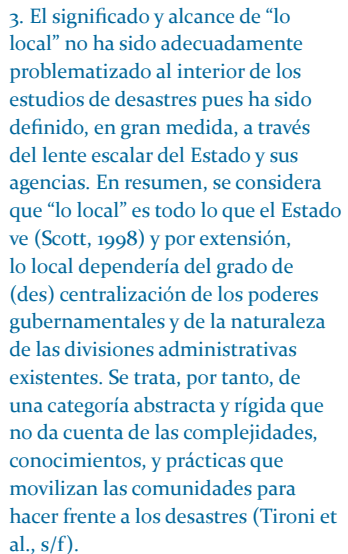


La técnica de recolección de datos utilizada fue la aplicación, grabación y transcripción anonimizada de entrevistas semiestructuradas a través de diferentes plataformas digitales durante los meses de mayo, junio y julio de 2020 y el registro de publicaciones en distintos medios digitales de prensa y redes sociales. Para el primer caso, los colaboradores fueron identificados mediante un muestreo selectivo de actores claves $(\mathrm{N}=12)$, uso de pauta semi-estructurada y consentimiento informado. El criterio de selección estuvo dado por la identificación de sujetos -cuyas voces y acciones se hacen presentes a lo largo del artículo a partir de la utilización de pseudónimos- que habían tomado algún rol en el contexto de la construcción del asentamiento y una posición con respecto a esta. Lo anterior fue posible gracias a una investigación previa (Acuña et. al., 2021) realizada en el territorio, lo que nos permitió contactar a los actores y actoras claves de este: desde quienes se hacían cargo de la dirigencia, quienes eran parte de la organización de las redes solidarias en el caso de quienes residen en el campamento, actores influyentes en las redes solidarias pero que no residen en el campamento, representantes de organizaciones sociales y/o políticas de la sociedad civil, un concejal de la comuna, familiares de residentes del campamento que fueron parte del proceso de organización de este, hasta residentes de las villas aledañas. Las entrevistas fueron acompañadas de notas de campo. Adicionalmente, una de las investigadoras del estudio efectuó trabajo en terreno como parte de una organización de ayuda solidaria vinculada con el territorio, aportando a la mirada y comprensión etnográfica del territorio, sus habitantes y organización sociopolítica. Para el segundo caso, se procedió a un registro de datos de plataformas digitales.

Finalmente, se efectuó un análisis de datos contextual en tres niveles: literal, interpretativo y reflexivo, siguiendo una perspectiva inductiva que consideró la emergencia de temáticas y conceptos directamente de los datos empíricos (Mason, 2017) -ver resumen en Tabla 1. La validez interna de los datos se propició por medio de la interpretación colectiva de los resultados entre el equipo de investigación.

\begin{tabular}{lllll}
$\begin{array}{l}\text { Aproximación } \\
\text { metodológica }\end{array}$ & $\begin{array}{l}\text { Técnicas de } \\
\text { recolección }\end{array}$ & $\begin{array}{l}\text { Instrumentos de } \\
\text { recolección }\end{array}$ & Materiales & Análisis \\
\hline & $\begin{array}{l}\text { Entrevistas semi- } \\
\text { estructuradas }\end{array}$ & $\begin{array}{l}\text { Pautas de } \\
\text { entrevistas semi- } \\
\text { estructuradas, }\end{array}$ & $\begin{array}{l}\text { Entrevistas }(\mathrm{N}=12), \\
\text { observaciones, } \\
\text { fotografias, fuentes }\end{array}$ & $\begin{array}{l}\text { Contextual de tres } \\
\text { niveles. }\end{array}$ \\
$\begin{array}{llll}\text { Estudio de caso } \\
\text { cualitativo. }\end{array}$ & $\begin{array}{l}\text { y observación } \\
\text { participante. }\end{array}$ & $\begin{array}{l}\text { nogistro de datos } \\
\text { primarias digitales. }\end{array}$ & \\
& & digitales. & & \\
\hline
\end{tabular}

Tabla 1. Resumen aproximación metodológica Fuente: Autoras, 2021.

\section{RESULTADOS}

\section{Contextualizando el Campamento Dignidad}

El "Campamento Dignidad" se ubica en la ribera norte de la Quebrada Macul, en la comuna de La Florida en Santiago de Chile (ver Figura 1). Esta zona fue epicentro de uno de los desastres más destructivos del país: el aluvión de 1993. Este último, arrasó con 307 viviendas, dañando a otras 5.610 , dejando a su paso 26 muertos, 85 heridos, 8 desaparecidos y 32.654 personas damnificadas (El Mercurio, 1993); y destruyendo las villas ubicadas en la ribera sur y norte de la Quebrada. Aunque tras el desastre se impidió la edificación en las riberas del cauce mediante la figura jurídica de "zona de restricción", desde diciembre de 2019 que el Campamento Dignidad alberga a más de 2.000 personas en una zona de riesgo de aluvión en plena pandemia de COVID-19.

El nombre con el cual se bautizó a este campamento alude directamente a la revuelta popular iniciada el 18 de octubre en el país donde "la demanda por la dignidad fue omnipresente en las protestas" (Brieba, 2020, p.32). A partir de lo anterior, entre diciembre del año 2019 y enero del 2020 varios grupos familiares que vivían como allegados ${ }^{4}$ en diferentes zonas de Santiago se tomaron el espacio aún conociendo los antecedentes históricos y geomorfológicos del territorio. Las familias reconocen que la ocupación de una zona de alta exposición a aluviones fue una estrategia para presionar a las autoridades a entregar soluciones habitacionales ante un abandono de años, como enfatiza una de las dirigentas del campamento: "[...] todos sabían que eso fue un
4. Expresión popular que describe una situación de carencia de vivienda propia. Se entiende por allegado(s) la persona, o núcleo folitir sin hijos, que vive en parte del sitio o en la casa de algún miembro de su familia extensa o de amigos. Las familias allegadas se calculan en aproximadamente 740.ooo, según MIDEPLAN, a través de diferentes encuestas Casen) 
aluvión ahí [...] como que creo que llega la desesperación de las personas y me incluyo, es como llegar, es como pedir a gritos, aparte que igual es un derecho [...]" (Magdalena, dirigenta).

En octubre del 2019, desencadenado por un aumento en las tarifas de transporte público en la capital, se desarrolló lo que fue conocido por los medios como "El Estallido Social" o "Revuelta de octubre". A partir de este hecho, durante meses, millones de personas residentes en Chile demostraron su descontento sobre la situación económica y política del país, poniendo énfasis en las desigualdades sociales y demandando derechos básicos como acceso a la salud, educación y vivienda. A raíz de lo anterior, desde finales del 2019 , ha habido un aumento en la cantidad de tomas de terrenos en nuestro país como expresión de luchas históricas por la vivienda. El grueso de los habitantes del Campamento Dignidad, son hijos de pobladores de la Toma Nasur de Peñalolén, iniciada en 1999. A dicha histórica toma se le entregaron distintas soluciones habitacionales, entre ellas las "casas chubi" . Sería de esas villas, en las que abunda el hacinamiento y allegamiento, de donde provienen buena parte de los habitantes del Campamento Dignidad; quienes por años han conformado comités de allegados en búsqueda de una solución que les permita acceder a una vivienda inserta en el entramado urbano, sin abandonar sus territorios ni redes de parentesco. Otro porcentaje de los habitantes, son personas de origen extranjero de comunas del sector poniente de la capital, quienes también han pasado por procesos extensos de postulación a vivienda definitiva, viendo frustrados sus proyectos por baches del mismo sistema. Por último, como la crisis sanitaria y económica ha provocado un aumento en el desempleo y la incapacidad de continuar pagando arriendos, vecinos del sector y comunas aledañas se han trasladado al campamento en busca de un techo:

"[...] yo soy un ejemplo de eso porque yo viví en la otra toma de NASUR, y se supone que iban a hacer otra toma en la misma toma de NASUR, se supone que iban a construir departamentos [...] y hasta el día de hoy en realidad no se ha hecho nada [...]" (Diana, dirigenta).

Hoy en día residen alrededor de600 familias. Adicionalmente, tiene una composición sociodemográfica heterogénea, con una significativa diversidad cultural. Cabe destacar, además, que la organización territorial de la toma se divide en 4 etapas representadas por dirigentas mujeres: "Porque ningún hombre ha tenido los cojones para hacerse cargo" (Sonia, dirigenta).

Al respecto, la participación femenina en dirigencias en las tomas de terreno tiene raíces históricas (Valdes \& Weinstein, 1993; Pérez, 2018) y estaría relacionada con los altos índices de jefatura femenina en hogares pobres, los cuales han ido en aumento en la última década: "[...] van a ser más dirigentas mujeres porque somos en el fondo las que vivimos con los hijos [...] el hombre es más fácil lavarse las manos y seguir su camino solo [...]" (Consuelo, hermana de residentes). Lo anterior refleja cómo las condiciones de las mujeres pobres en Chile, y particularmente en el campamento, son de alta vulnerabilidad ya que proveen de trabajo reproductivo, cuidado no remunerado, ingresos económicos para sus familias (Pérez, 2018); además de gestionar techo y salud, y en el caso de las dirigentas, las redes solidarias internas y externas del territorio.

Considerando los orígenes y condiciones de vida del Campamento Dignidad, a continuación, damos cuenta de las formas de organización comunitaria y las estrategias para hacer frente a las amenazas de desastres presentes en el territorio, con el objetivo de ilustrar cómo en asentamientos informales las capacidades locales se articulan estratégicamente para dar seguridad a sus vidas.

\section{Amenazas y organización comunitaria}

A modo de dar cuenta de nuestros resultados (ver Tabla 2), a continuación, describimos etnográficamente qué es lo que se constituye como amenaza de desastre en el Campamento Dignidad, como también sus formas particulares de gestión comunitaria. A partir del análisis de las entrevistas y los focos de prevención de riesgo por parte de las dirigentas y residentes del campamento, logramos identificar múltiples amenazas: pandemia, aluvión, inseguridad alimentaria, escasez hídrica, inundaciones e incendios.

Las capacidades de organización comunitaria frente a las múltiples amenazas se constituyen tanto por redes solidarias internas como externas al territorio, que articulan distintos tipos de estrategias que buscan dignificar la vida de los residentes del campamento, basadas por una parte, en el cuidado y colaboración, y por otra, en el apoyo de emergencia a través de distintos tipos de conocimientos y recursos solidarios. 


\begin{tabular}{|c|c|c|}
\hline Amenazas múltiples & Organización comunitaria & Estrategias \\
\hline \multicolumn{3}{|l|}{ Pandemia } \\
\hline $\begin{array}{l}\text { Inseguridad alimentaria, } \\
\text { Incendios }\end{array}$ & $\begin{array}{l}\text { Interna: división del campamento } \\
\text { por etapas, vocería, turnos de } \\
\text { trabajo. }\end{array}$ & $\begin{array}{l}\text { Redes solidarias internas: } \\
\text { cuidado y colaboración. }\end{array}$ \\
\hline $\begin{array}{l}\text { Inundaciones } \\
\text { Aluvión }\end{array}$ & $\begin{array}{l}\text { Externa: organizaciones político- } \\
\text { territoriales y voluntarias. }\end{array}$ & $\begin{array}{l}\text { Redes solidarias externas: } \\
\text { recursos (humanos y materiales), } \\
\text { conocimiento local y experto en } \\
\text { emergencias. }\end{array}$ \\
\hline Escasez hídrica & & \\
\hline
\end{tabular}

Tabla 2. Sintesis de resultados

Fuente: Autoras, 2021.

A raíz de la pandemia COVID-19, los lineamientos que el gobierno de Chile adoptó desde marzo de 2020 respecto al manejo de esta, han consistido en el lavado constante de manos, uso de mascarilla, mantenimiento del distanciamiento social, evitar aglomeraciones y cumplir con la normativa del Ministerio de Salud. No obstante, estos no han sido coherentes con las posibilidades y preocupaciones que viven los residentes del campamento a diario. Como menciona una voluntaria que presta ayuda a la toma, "la cuarentena se vive de otra forma": puesto que la gente circula por las calles de tierra, casi no usa mascarillas, se reúne en las ollas comunes o las asambleas exponiéndose al virus, porque así se logra evitar el hambre. Esto no sólo incrementa la posibilidad de contagiarse, sino que muestra además cuán críticas pueden ser las consecuencias de la desigualdad estructural en asentamientos informales donde no está asegurado el acceso a la vivienda digna (Pérez, 2018).

Según el Banco Mundial (2020), las personas que conviven con la pobreza urbana tienen mayor riesgo de contraer coronavirus que aquellas personas con mayores ingresos. En parte, debido a que dada las características informales de la fuerza laboral, las regulaciones son menores, al igual que los salarios y las prestaciones sociales, habiendo en consecuencia una mayor desprotección social. Además, como los asentamientos informales suelen estar marginados de los servicios básicos, existe una falta de sanitización e ingesta de agua potable, impactando negativamente en la salud de las personas [...]" (Banco Mundial, 2020)

En la práctica, las consecuencias de la pandemia se hicieron evidentes a partir del mes de mayo de 2020, donde se evidenciaron en el desempleo y por ende en la inseguridad alimentaria al interior del Campamento Dignidad. Esto tuvo su origen en el endurecimiento de las medidas restrictivas de circulación y en la deficiente asistencia económica de emergencia por parte del gobierno, la cual no alcanzó a suplir las necesidades alimentarias básicas de la población (para una visión más global de este fenómeno, revisar Gil et al., 2021).

Pero en este contexto, el virus y el hambre no son las únicas amenazas. Las características precarias de las viviendas autoconstruidas, la carencia de servicios básicos y el emplazamiento de estas hacen que las amenazas se multipliquen. A modo de ejemplo, el 3 de octubre de 2020 ocurrió un incendio que dañó seis viviendas y dejó 22 damnificados fruto de las condiciones materiales y estructurales de habitabilidad del espacio y hacinamiento. Asimismo, durante el invierno del mismo año hubo un aumento inesperado de las precipitaciones en la ciudad de Santiago, lo cual provocó que muchas de las viviendas quedaran inhabitables debido a las inundaciones. Recientemente, a finales del mes de enero de 2021 , se pronosticó un sistema frontal con altas intensidades de precipitaciones y características meteorológicas que no se evidenciaban desde el desastre de 1993. Esto significó la declaración de alerta preventiva por riesgo de aluviones para todas las quebradas del piedemonte de Santiago y en particular, para la Quebrada de Macul.

Las respuestas ante estas múltiples amenazas de desastres, en el Campamento Dignidad, son usualmente gestionadas a partir de organizaciones comunitarias que responden a distintos objetivos que terminan articulando diversas estrategias en formas de redes de solidaridad externas e internas al territorio; echando mano a la herencia histórica de los campamentos como espacios estructurados bajo una organización política articulada en torno a la demanda de derechos urbanos, como fue especialmente durante los años de la Unidad Popular (Pérez \& Palma, 2021; Castells, 1973; Giusti, 1973; Pastrana \& Threlfall, 1974). 
Por un lado, la organización comunitaria al interior del Campamento Dignidad se configura a partir de una división socio-territorial en cuatro etapas, cada una liderada por una dirigenta,y una vocera que se encarga de las comunicaciones con organizaciones externas e instituciones estatales. Esta organización parece estar basada en prácticas comúnmente asociadas a liderazgos femeninos -como el cuidado- y en la ayuda comunitaria y diversificación equitativa del trabajo femenino. En el caso de los hombres, estos colaboran en momentos de emergencia mediante el apoyo en situaciones específicas como en la autoconstrucción de las viviendas, guardias en momentos de ocupación de terrenos y alertas de riesgo de aluvión.

Por otro lado, la organización comunitaria externa al territorio está constituida por distintas agrupaciones solidarias -El hambre no espera, Red la Olla, Movimiento por la Defensa del Agua, la Tierra y el Medio Ambiente (MODATIMA), Escuelita Dignidad etapa 4, entre otros ${ }^{6}-$, y político-territoriales -Brigada de Emergencia Alto La Florida (BEAF) y Asamblea Territorial Aluvión (ATA), entre otras-. Las primeras, también están constituidas por liderazgos femeninos, cuyas organizaciones priorizan diferentes aspectos de las múltiples amenazas que se viven en el Campamento Dignidad: inseguridad alimentaria, vulneración del derecho al agua y del derecho a la salud. Estas organizaciones solidarias se gestaron de manera autónoma a partir de iniciativas individuales para colaborar frente a las necesidades del territorio. Las segundas están constituidas por liderazgos masculinos y se basan en la identificación político-territorial y en el conocimiento histórico-local de la quebrada.

Ambas formas organizacionales articulan estrategias en el territorio a través de las cuatro dirigentas y la vocera, que son las encargadas de vincular las ayudas externas con las necesidades de los y las vecinas del campamento. Es en momentos de emergencia y de abandono estatal, en las que se hace evidente la necesidad de una colaboración entre todas estas formas de organización comunitaria, lo que se ve reflejado durante la alerta preventiva de aluvión del viernes 29 y sábado 30 de enero 2021.

En el contexto de la pandemia por coronavirus fueron las dirigentas quienes tomaron el rol cuidador, en forma de fiscalización y educación para prevenir los contagios, siendo la estrategia preponderante durante los primeros meses del 2020:

"[...] a los chiquillos se les ha repartido mascarilla, cuando ha llegado alcohol gel, alcohol gel [...] también ayudándolos con el sentido de que, acordarlos porque muchos trabajando se les olvida lavarse las manos [...]" (Diana, dirigenta etapa 1).

En el caso de diagnósticos de coronavirus positivos, las medidas de cuarentena obligatoria han sido sólo posibles gracias a las acciones de liderazgo femenino que prevalecen en el Campamento Dignidad en tanto, las dirigentas son capaces de posibilitarlas a través del conocimiento de las necesidades particulares de los vecinos y de la gestión de la ayuda material proveniente de redes solidarias externas. A modo de ejemplo, uno de los casos en los que una familia se contagió de coronavirus, la dirigenta de su etapa posibilitó la cuarentena a través del cuidado:

"les hice una caja de mercadería como tenían guaguita les llevé pañales, alimentos no perecibles y me preocupaba de ir a comprarles el pan al negocio y ir a dejarlo a la puerta [...] le pasé cloro, le pasé desinfectante, alcohol gel, guantes, mascarilla" (Sonia, dirigenta).

Otra forma en la que se buscó prevenir los efectos de la pandemia en el campamento fue a través de la toma de $\mathrm{PCR}^{7}$ en el territorio, lo que se dio en meses en los que todavía era difícil encontrar operativos de "búsqueda activa" de casos de COVID-19. Este evento fue gestionado autónomamente a partir de una asistente social de uno de los $\mathrm{CESFAM}^{8}$ de la comuna.

En miras de la escasez de agua en el contexto de un asentamiento informal y de la pandemia, también fue necesario gestionar colaboraciones de organizaciones externas al territorio. En este escenario, se hizo presente MODATIMA, a través de una de sus integrantes-, quien generó el vínculo con las dirigentas del campamento para hacer frente a la ausencia de recursos hídricos en el territorio, a la vez que buscó generar conciencia entre la correlación existente entre el derecho a la vivienda y el derecho al agua:

"El eslogan que estamos haciendo para la campaña del agua para la Toma que es que vulnerar el derecho a la vivienda es vulnerar el derecho al agua. Y por lo tanto, también vulnerar el derecho a la salud [...]" ( Paz, integrante MODATIMA).
6. Desde el inicio de la ocupación del territorio diversas organizaciones comunitarias externas e internas han ido consolidándose y colaborando con el Campamento Dignidad. Existen una serie de colectivos que participan en distintos momentos del tiempo a propósito de las necesidades que van emergiendo. Para el propósito de esta investigación, sólo se consideraron las organizaciones más activas durante duró esta investigación (2019-2021).

7. Polymerase Chain Reaction (Reacción en cadena de la polimerasa) examen utilizado para diagnosticar diferentes enfermedades, entre ellas el SARS-CoV-2.

8. Centro de Salud Familiar 
Las estrategias utilizadas por las dirigentas son las que logran articular las ayudas externas al territorio con las necesidades de los residentes del campamento. Esto es lo que permite controlar el virus desde la posibilidad de realización de cuarentenas de grupos familiares enteros en condiciones de extrema vulnerabilidad y pobreza, hasta contar con el recurso básico del agua para higienización y consumo humano.

Asimismo, fruto de las consecuencias de la pandemia en la inseguridad alimentaria, se implementó la olla común -como estrategia histórica que de igual forma descansa en liderazgos femeninos- activando las redes solidarias para hacer frente al hambre. Esto se realizó a través del vínculo entre las dirigentas y una voluntaria de la organización de la sociedad civil "El Hambre no Espera" que su origen se encuentra en el diagnóstico de falta de recursos alimentarios en el Campamento Dignidad: "comenzamos con el tema de la pandemia donde evidentemente los recursos económicos no estaban para la gente [...] se empieza a hacer la olla común todos los días" (Jimena). Estas redes externas se encargaban de abastecer con los insumos necesarios a las redes internas que son preferentemente gestionadas por mujeres y que funcionan gracias a la división del trabajo: "nos llegan raciones de comida, y las mujeres nos encargamos de cocinar, entonces se hacen como turnos [...]" (Camila, residente del Campamento Dignidad).

Estas redes se logran configurar gracias a los liderazgos femeninos de cada una de ellas, que se basan en estrategias de cuidado mutuo, que van desde relaciones de parentesco a solidaridad vecinal. Estas estrategias parecen emerger y replicar lógicas de cuidado que son comprendidas por parte de las dirigentas como propias de un rol materno: "Yo soy la mamá como de casi 800 personas se podría decir, [...] Tenemos 192 niños, más los adultos, guagüitas que han nacido aquí, yo soy la mamá de todos ellos" (Sonia, dirigenta).

Esto se refleja también en la preocupación constante de las dirigentas hacia los niños y niñas del campamento, ante las diversas situaciones de riesgo. Ellos son en muchos casos una de las preocupaciones principales cuando se ponen en el escenario de un posible aluvión, de desalojo, 0 incluso ante la necesidad de aprendizaje y cuidado cotidiano de estos en el campamento en el contexto de la pandemia. Estas preocupaciones constantes se encuentran presentes en los discursos y relatos de las dirigentas sobre la vida cotidiana en el campamento:

"el otro día estuve con dos niños de la toma [...] tenía leche, tenía yogurt, y al otro día junté otros tres más [...] así no andan tanto afuera, en el barro, la humedad, el frío [...] hemos querido hacer como tipo escuelita [...] está la disposición de hacerles algo pero nos ha costado un mundo igual formar algo como para hacer una escuelita" (Magdalena, dirigenta).

A partir de la necesidad identificada por las dirigentas y voluntarias, con respecto a la situación escolar de los niños y niñas durante la pandemia, se logró articular una red solidaria de diferentes "escuelitas" en distintas etapas del campamento (dignidad, popular etapa 3 y dignidad sector 4).

Otra de las preocupaciones constantes de los residentes, sobre todo al comienzo de la ocupación de los terrenos, tenía que ver con el posible desalojo y el destino de los niños. Si es que fuese necesario, el plan de acción consistía en que las dirigentas se harían cargo del cuidado de los niños y niñas: "Se suponía que si en caso de desalojo mi casa iba a ser como la guardería, donde iban a llegar a todos los niños" (Diana, dirigenta). Esta estrategia, es otra forma de reflejar el rol materno tomado por las dirigentas en el territorio.

Por otro lado, la amenaza de incendio es algo permanente en el contexto de asentamientos informales, cuyas condiciones de habitabilidad no son óptimas debido a los materiales de construcción de las viviendas, el hacinamiento y las formas irregulares por las cuales se abastecen del recurso eléctrico. Desde el comienzo de la ocupación de terreno, las dirigentas han mantenido una preocupación con respecto a la posibilidad de un incendio: "un incendio que la gente está toda colgada, nada, un 'chispazo' se puede quemar una casa, y no se va a quemar una [...] (Diana, dirigenta).

Luego del incendio (ver Figura 2), se activaron una vez más las redes solidarias ya articuladas en el territorio generando campañas para la recolección de materiales de construcción y ropa, entre otros. En este momento de emergencia, incluso se hizo presente la municipalidad a través de la entrega de mediaguas ${ }^{9}$ a las familias afectadas por el incendio. No obstante lo anterior, los liderazgos femeninos que poseen conocimiento del territorio y de la vida cotidiana al interior del 
campamento, identificaban una falencia en las estrategias de colaboración y GRD tanto solidaria como estatal debido a que no tenían un foco en la prevención de eventos futuros: "[...] Ahora ¿sabes lo que quiero hacer con lo visto? Es hacer una campaña de extintores y luces de seguridad" (Jimena, voluntaria de la organización "El Hambre No Espera").

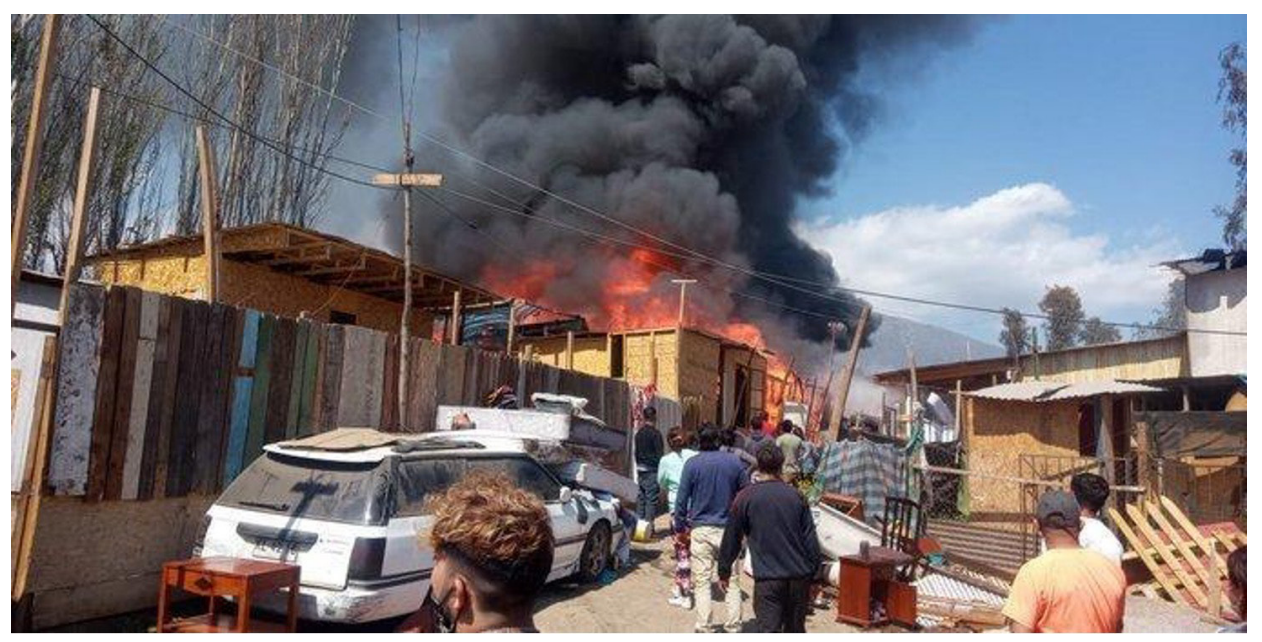

Figura 2. Incendio en Campamento Dignidad Autora: Residente del Campamento Dignidad, 2021.

Otra de las amenazas que devienen de la fragilidad de los materiales y de la ubicación del campamento, ocurre cada vez que hay precipitaciones en el territorio, causando inundaciones que tienen como consecuencia la inhabitabilidad de las viviendas y el riesgo de aluvión. Para el primer caso, se han activado las redes solidarias a través de las organizaciones comunitarias en varias ocasiones desde la constitución del campamento, a través de campañas de recaudación de fondos para la compra de materiales de construcción (Zinc) que protejan las viviendas de las lluvias.

Con respecto al riesgo de aluvión, existen organizaciones territoriales y políticas que buscan colaborar con el Campamento Dignidad frente a este. Estas organizaciones, como la ATA, tienen a la base de su interés de colaboración, su experiencia en el territorio ya que muchos de sus integrantes vivieron el desastre de 1993 y buscan contribuir con su conocimiento local en conjunto con los habitantes del campamento, como expresa una de las dirigentas:

"[...] acá todos los vecinos saben, ponle los 'chiquillos' de MODATIMA [...] los de ATA, nos hicieron un video para el... cuando prendimos las velitas acá y todo [...] y yo lo mandé a los grupos de WhatsApp para que los delegados lo vieran y todo, así que están todos informados, acá todos saben del tema del aluvión" (Daniela, dirigenta etapa 1).

A pesar de que cada organización comunitaria prioriza una de las amenazas presentes en el territorio en distintas circunstancias, en los momentos de mayor emergencia, todas las organizaciones y redes ya articuladas en el territorio se cohesionan para hacer frente a la amenaza.

Durante la última semana de enero, la dirección meteorológica de Chile pronosticó condiciones climáticas que no se repetían desde 1993, traduciéndose, por tanto, en un alto riesgo de aluvión para el territorio en el que se ubica el Campamento Dignidad. Si bien esta situación era conocida por las autoridades competentes, no se dio ningún tipo de alerta oficial y directa a la población expuesta, solamente se publicaron infografías en redes sociales (ver Figura 3) -de acuerdo al manejo institucional de las emergencias en Chile, las instituciones a cargo de este tipo de alertas y evacuaciones preventivas son ONEMI ${ }^{10}$, SERNAGEOMIN ${ }^{11}$ y las municipalidades. En este escenario, el jueves 28 de enero, los vecinos y vecinas del campamento comenzaron a solicitar información sobre qué acciones seguir a las redes articuladas del territorio; principalmente en torno a la ATA y a la BEAF. Fue tal la sensación de desamparo, que estas entidades acudieron a la institucionalidad a través de la academia, es decir, al centro de investigación donde trabajamos las autoras de este artículo (CIGIDEN), para solicitar apoyo en la gestión de esta emergencia.
10. Oficina Nacional de Emergencia del Ministerio del Interior.

11. Servicio Nacional de Geología y Minería de Chile. 


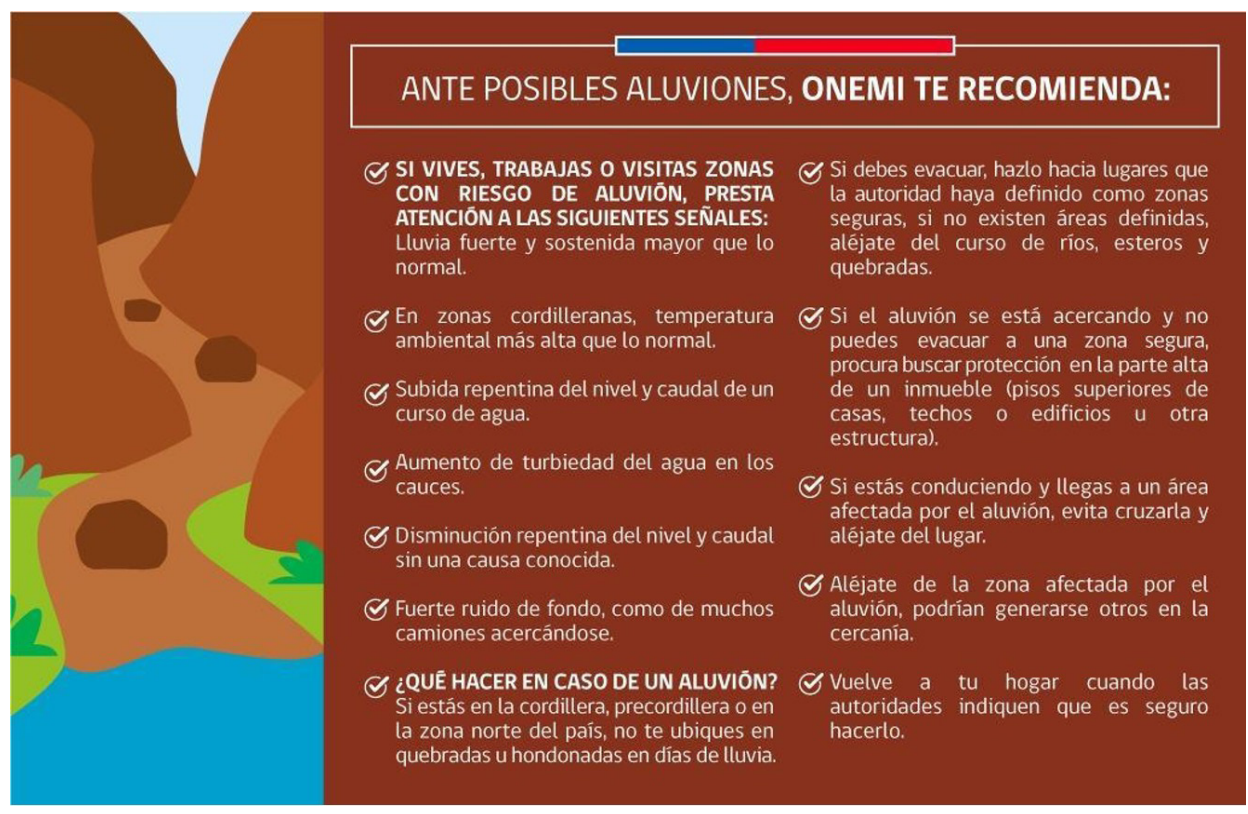

Figura 3. Infografía ante posibles aluviones

Fuente: Oficina Nacional de Emergencias, ONEMI, 2021

Al día siguiente, durante la jornada del viernes 29 , los vecinos y vecinas del campamento se mantuvieron en estado de alerta formando guardias en las diferentes etapas solamente con las directrices que desde CIGIDEN se les había entregado -en base a recomendaciones generales sobre la evacuación en el territorio, tomando en cuenta el conocimiento sobre la Quebrada de Macul y otras quebradas aledañas. Por otro parte, la BEAF, a pesar de no pertenecer al sistema de protección civil formal, fue tomando protagonismo. Recién en la tarde de ese día la municipalidad de La Florida se contactó con los liderazgos de la brigada para armar un plan de monitoreo del cauce y de las obras de mitigación en conjunto con personal municipal y Carabineros de Chile. Sin embargo, la dirigencia del Campamento Dignidad no fue informada del plan por parte de la municipalidad de manera formal.

El sábado 30 por la mañana las lluvias intensas continuaron y la alerta preventiva por riesgo de aluvión se mantuvo. Por la tarde, la preocupación y sensación de urgencia volvió al campamento; recién ese día a las 9 de la noche desde el municipio de La Florida se comunicaron por primera vez con la vocera del campamento: "Chiquillos les comunico que me acaba de llamar Rodolfo Carter, avisándome que tenemos que evacuar diciendo que hay un posible alud [...] (Andrea ${ }^{12}$, Vocera, 20:54). La evacuación se llevó a cabo alrededor de las 23:00 horas de esa noche, y al día siguiente por la mañana, la vocera del campamento comenzó a gestionar ayuda para poder reparar los techos del campamento, anticipándose a la vuelta de las familias al lugar. Por su parte, un grupo de personas decidieron no abandonar el terreno en el que se encontraban sus viviendas por miedo al desalojo.

Las diversas gestiones locales comunitarias descritas en este apartado se hacen necesarias para enfrentar las distintas amenazas ante la ausencia de entidades estatales en este asentamiento informal. Esta experiencia vuelve evidente cómo la articulación entre las redes internas y externas de gestión y solidaridad en el campamento se utilizan cuando falla la intervención institucional, responsabilizando a las comunidades de autogestionar el riesgo de desastres.

\section{DISCUSIÓN Y CONCLUSIONES}

A pesar de las limitaciones que tienen los estudios de carácter cualitativo y que tiene la presente investigación, al encontrarse situada en un territorio con características tan específicas como la Quebrada de Macul y en un contexto completamente extraordinario debido a la pandemia, El Campamento Dignidad, como caso de estudio, ha sido un fiel reflejo del desacoplamiento tanto de las políticas de planificación territorial como de las políticas de gestión del riesgo de desastres, con respecto de los asentamientos informales y sus formas de organización política interna. Esto ha sido evidenciado extensamente por la literatura de ciencias sociales existente sobre reducción
12. Mensaje de Audio grupo

"Comunicación y prevención", 30 de enero de 2021. 
del riesgo de desastres, poniendo énfasis en la crítica a la forma en que las políticas de riesgo y planificación comprenden el concepto de resiliencia.

Lo descrito en este artículo busca evidenciar que en el contexto de asentamientos informales expuestos a múltiples amenazas, la población hace frente a estas a partir de una gestión y articulación constante de redes y recursos según la urgencia que ellos mismos les entregan dependiendo de las especificidad de las condiciones climáticas, sanitarias y, económicas, entre otras. Cuando el concepto de resiliencia se construye prestando atención a las voces subalternas y excluidas de las políticas de gestión del riesgo que provienen de parte de la institucionalidad, se debe considerar que las comunidades no son estables y que están lejos de ser despolitizadas. En el caso de los campamentos, estos son espacios articulados en base a una lucha histórica por la vivienda caracterizada por la categoría moral de dignidad, la cual busca transformar las condiciones de vulnerabilidad que son inherentes al riesgo.

Uno de los elementos fundamentales para la conformación de estrategias al interior del Campamento Dignidad, de la mano de los liderazgos femeninos y su articulación con redes solidarias externas al territorio, tiene que ver con la gestión comunitaria, que tienen como eje central principios éticos como el cuidado y la solidaridad. Esto se vuelve evidente a través del discurso de las dirigentas, en el que se autodefinen como "mamás" de los y las vecinas del campamento, poniendo especial énfasis en la gestión de redes alimentarias, en el cuidado de los niños y niñas y en el conocimiento de las necesidades específicas de los diferentes miembros de su comunidad.

Las relaciones existentes dentro del campamento invitan también a problematizar desde la literatura del cuidado y género, las nociones de parentesco y "hogar" que se manejan en las políticas estatales de vivienda y de GRD ya que en muchos casos es evidente cómo los límites de los hogares como núcleos familiares radicados en viviendas particulares, se desdibujan al ser parte de un entramado social que tiene líderes que se posicionan como "cuidadoras". Tomando el argumento de Clara Han, sobre que en espacios vulnerables se "forman relaciones domésticas que, además, mitigan las fuerzas de la precariedad económica" (Han, 2012), bajo las condiciones del Campamento Dignidad, podemos afirmar que estas formas de relación -y su componente ético, de cuidado y de derechos, vinculados a la noción de dignidad- exigen expandir la comprensión de resiliencia hacia una transformación de los regímenes del riesgo.

Para finalizar, esta investigación deja abierta dos interrogantes claves para la futura investigación de GRD en asentamientos informales. En primera instancia, dado que la ausencia del acceso a la vivienda digna está íntimamente relacionada con las amenazas de distintos tipos de desastres y las estrategias comunitarias para enfrentarlos; una GRD en asentamientos informales que sea robusta, debe estimular políticas de desarrollo que se hagan cargo del problema habitacional, considerando las formas particulares de relación y organización, y por ende las categorías tradicionales de hogar. En segunda instancia, hemos visto que no sólo el problema de la vivienda está íntimamente relacionado con desigualdades de género sino también con la propia gestión del riesgo en asentamientos informales. La literatura, si bien ha hecho el hincapié en comprender los recursos y fortalezas de género con el mismo entusiasmo con que se estudian su vulnerabilidad en situaciones de desastres (Enarson et al., 2018), la "feminización de la responsabilidad" puede terminar reforzando las relaciones de género asimétricas existentes si es que no se rompe con los estereotipos y roles de género (Ramirez \& Becerril, 2021). De este modo, si las iniciativas de GRD tienen realmente como uno de sus objetivos reducir la vulnerabilidad de las mujeres, deben buscar el modo de centrarse explícitamente en las causas fundamentales de esta vulnerabilidad y diseñar programas sensibles al género, a la medida de sus intereses, necesidades y expandiendo sus capacidades (Ramirez \& Becerril, 2021) y poniendo el foco en la reducción de las desigualdades (Bradshaw, 2015).

\section{REFERENCIAS}

Acuña, V., Juzam, L., Tironi, M. \& Valdivieso, S. (24 de julio de 2020). La pandemia en Toma Dignidad: re-pensando la Gestión del Riesgo de Desastres en asentamientos informales. CIPER. https://bit. ly/3fZEYxc

Acuña, V., Roldán, F., Tironi, M. \& Juzam, L. (2021). The Geo-social Model: A Transdisciplinary Approach to Flow-type Landslide Analysis and Prevention. Sustainability, 13(5), 2501.

Aldrich, D.P. (2008). The crucial role of civil society in disaster recovery and Japan's preparedness for emergencies. Japan aktuell, 3(2008), 81-96. 
Allen, A., Zilbert Soto, L., Wesely, J., Belkow, T., Ferro, V., Lambert, R., Langdown, I., \& Samanamú, A. (2018). From state agencies to ordinary citizens: Reframing risk-mitigation investments and their impact to disrupt urban risk traps in Lima, Peru. Environment and Urbanization, 29(2), 477-502

Allen, A., Sarmiento, J.P. \& Sandoval, V. (2020). Los Estudios Latinoamericanos de Reducción del Riesgo de Desastres en el Contexto de la Pandemia del COVID-19. Revista de Estudios Latinoamericanos sobre Reducción del Riesgo de Desastres REDER, 4(2), 1-6. http://revistareder.com/ handle-0719-8477-2020-066

Araujo, K., Poblete, P., Montiglio, M.A.N., \& Echenique, G.V. (2019). Hilos tensados. Para leer el octubre chileno. USACH.

Biskupovic, C. (2019). Desnaturalizar lo natural. El aluvión de la Quebrada de Macul como proceso social en Santiago de Chile. Antropologías del Sur, 12, 107-128.

Boano, C. (2017). Pathways towards the resilient city: Presupposition of equality and active justice in Bangkok, Thailand. In A. Allen, L. Griffin, \& C. Johnson (Eds.), Environmental justice and urban resilience in the Global South (pp. 137-152). Palgrave Macmillan.

Bradshaw, S. (2015). Engendering development and disasters. Disasters, 39(s1), s54-s75.

Brieba, D. (2020). El estallido social en Chile desde el igualitarismo relacional de Elizabeth Anderson. Revista de Sociología, 35(1), 31-42.

Brightman, R. (1995). Forget Culture: Replacement, Transcendence, Relexification. Cultural Anthropology, 10(4), 509-546.

Brown, K., \& Westaway, E. (2011). Agency, capacity, and resilience to environmental change: lessons from human development, well-being, and disasters. Annual review of environment and resources, $36,321-342$.

Brown, K. (2012). Policy discourses of resilience. In M. Pelling, D. Manuel-Navarrete, \& M. Redclift (Eds.), Climate change and the crisis of capitalism: A chance to reclaim self, society and nature (p. 37). Routledge.

Brown, K. (2014). Global environmental change I: A social turn for resilience? Progress in Human Geography, 38(1), 107-117.

Bull-Kamanga, L., Diagne, K., Lavell, A., Leon, E., Lerise, F., MacGregor, H., ... \& Yitambe, A. (2003). From everyday hazards to disasters: the accumulation of risk in urban areas. Environment and Urbanization, 15(1), 193-204.

Castells, M. (1973). Movimiento de Pobladores y Lucha de Clases. EURE, 3(7), 9-36.

Cote, M., \& Nightingale, A.J. (2012). Resilience thinking meets social theory: situating social change in socio-ecological systems (SES) research. Progress in human geography, 36(4), 475-489.

Cretney, R. (2014). Resilience for whom? Emerging critical geographies of socio-ecological resilience. Geography Compass, 8(9), 627-640.

Deeming, H., Fordham, M., Kuhlicke, C., Pedoth, L., Schneiderbauer, S., \& Shreve, C. (Eds.). (2019). Framing community disaster resilience. John Wiley \& Sons.

Enarson, E., Fothergill, A., \& Peek, L. (2018). Gender and disaster: Foundations and new directions for research and practice. In Handbook of disaster research (pp. 205-223). Springer.

El derrumbe del cielo (5 de septiembre de 1993). El Mercurio.

Elmqvist, T. (2014). Urban resilience thinking. Solutions, 5(5), 26-30.

Evans, J.P. (2011). Resilience, ecology, and adaptation in the experimental city. Transactions of the Institute of British Geographers, 36, 223-237.

Fenton, P., \& Gustafsson, S. (2017). Moving from high-level words to local action-governance for urban sustainability in municipalities. Current opinion in environmental sustainability, 26, 129-133.

Flores, P., Juzam, L., Miranda, F., \& Vergara, F. (2019). Modelo de gestión del riesgo de desastres en campamentos. Serie de Policy Papers CIGIDEN.

Fortun, K. (2001). Advocacy after Bhopal: Environmentalism, Disaster, New Global Orders. University of Chicago Press.

Fuentealba, R. \& Verrest, H. (2020). Disrupting Risk Governance? A Post-Disaster Politics of Inclusion in the Urban Margins. Urban Planning, 5(3), 274-287.

Gil, D., Dominguez, P., Undurraga, E. A., \& Valenzuela, E. (2021). The Socioeconomic Impact of COVID-19 in Urban Informal Settlements. MedRxiv. https://doi.org/10.1101/2021.01.16.21249935 
Giusti, J. (1973). Organización y Participación Popular En Chile: El Mito Del “Hombre Marginal." Santiago de Chile: Ediciones FLACSO.

Gupta, A. \& Ferguson, J. (1992). “Beyond Culture”: Space, Identity, and the Politics of Difference. Cultural Anthropology, 7(1), 6-23.

Han, C. (2012). Life in debt: Times of care and violence in neoliberal Chile. University of California Press.

Herrle, P., Ley, A., \& Fokdal, J. (2015). From Local Action to Global Networks: Housing the Urban Poor. Farnham: Routledge.

Mina, H., Howitt, R. \& Miller, F. (2015). Postcolonial Vulnerability and Institutional Capacity Deficits in Post-Disaster Recovery and Reconstruction: Insights from Wutai Rukai Experiences of Typhoon Morakot. Human Organization, 74(4), 308-318

Joseph, J. (2013). Resilience as embedded neoliberalism: a governmentality approach. Resilience, 1(1), 38-52.

Kaika, M. (2017). 'Don't call me resilient again!': The New Urban Agenda as immunology ... or ... what happens when communities refuse to be vaccinated with 'smart cities' and indicators. Environment $\mathcal{E}$ Urbanization, 29(1), 89-102. https://doi.org/10.1177/0956247816684763

Keck, M., \& Sakdapolrak, P. (2013). What is social resilience? Lessons learned and ways forward. Erdkunde, 5-19.

Lavell, A. (2004). La red de Estudios Sociales en Prevención de Desastres en América Latina, LA RED: Antecedentes, formación y contribución al desarrollo de los conceptos, estudios y la práctica en el tema de los riesgos y desastres en América latina: 1980-2004. Panamá: Red de Estudios Sociales en Prevención de Desastres en América Latina, noviembre. [Documento en línea]. https://www. desenredando.org/public/varios/2004/LARED-AFCDCEPTRDAM/LARED-AFCDCEPTRDAM_nov26-2004.pdf

Lavell, A., \& Maskrey, A. (2014). The future of disaster risk management. Environmental Hazards, 13(4), 267-280

Nkosi, V., Haman, T., Naicker, N., \& Mathee, A. (2019). Overcrowding and health in two impoverished suburbs of Johannesburg, South Africa. BMC public health, 19(1), 1358.

Manyena, B., O’Brien, G., O’Keefe, P., \& Rose, J. (2011). Disaster resilience: a bounce back or bounce forward ability? Local Environment: The International Journal of Justice and Sustainability, 16(5), 417-424.

Marulanda, M.C., Cardona, O.D., \&Barbat, A.H. (2010). Revealing the socioeconomic impact of small disasters in Colombia using the DesInventar database. Disasters, 34(2), 552-570.

MacKinnon, D., \& Derickson, K.D. (2013). From resilience to resourcefulness: A critique of resilience policy and activism. Progress in human geography, 37(2), 253-270.

Mason, J. (2017). Qualitative researching. Sage.

Matyas, D., \& Pelling, M. (2014). Positioning resilience for 2015: The role of resistance, incremental adjustment and transformation in disaster risk management policy. Disasters, 39 (SI), S1-S18.

McLean, H., \& Ewart, J. (2015). Political communication in disasters: A question of relationships. Culture Unbound, 7(3), 512-523.

Ministerio de Vivienda y Urbanismo MINVU. (2019). Catastro Nacional de Campamentos MINVU 2019. https://storymaps.arcgis.com/stories/dfeiferafd334ec79of879e736a5af5e

Mitlin, D. (27 de marzo de 2020). Dealing with COVID-19 in the towns and cities of the global South. International Institute for Environment and Development. https://www.iied.org/ dealing-covid-19-towns-cities-global-south

Mitra, S., Mulligan, J., Schilling, J., Harper, J., Vivekananda, J. \& Krause, L. (2017). Developing risk or resilience? Effects of slum upgrading on the social contract and social cohesion in Kibera, Nairobi. Environment \& Urbanization, 29(1), 103-122.

MacKinnon, D., \& Derickson, K.D. (2012). From resilience to resourcefulness: A critique of resilience policy and activism. Progress in Human Geography, 37(2), 253-270.

Oliver-Smith, A. (1999). What is a Disaster? Anthropological Perspectives on a Persistent Question. In Oliver-Smith, A. \& Hoffman, S. (Eds.), The Angry Earth: Disaster in Anthropological Perspective (pp.18-34). Routledge. 
Ortiz, C., \& Di Virgilio, M.M. (2020). Laboratorios de Vivienda (LAVs): Asentamientos precarios y vivienda social: impactos del covid-19 y respuestas. Urban Housing Platform Hub.

Paton, D. (2006). Disaster resilience: building capacity to co-exist with natural hazards and their consequences. Disaster resilience: An integrated approach, 3-10.

Paul, B.K. (2011). Environmental hazards and disasters: contexts, perspectives and management. John Wiley \& Sons.

Pastrana, E., \& Threlfall, M. (1974). Pan, Techo y Poder. El Movimiento de Pobladores En Chile (19701973). Ediciones Siap-Planteos.

Pérez, M. (2018). Toward a Life with Dignity: Housing Struggles and New Political Horizons in Urban Chile. American Ethnologist, 45(4), 508-520.

Pérez, M. \& Palma, C. (2021). El retorno de los campamentos: Cinco mitos que oscurecen el debate. Ciper académico/análisis. https://www.ciperchile.cl/2021/04/28/ el-retorno-de-los-campamentos-cinco-mitos-que-oscurecen-el-debate/

Pizzo, B. (2015). Problematizing resilience: Implications for planning theory and practice. Cities, 43, 133-140.

Ramírez, C. \& Becerril, H. (2021). Prevención del Riesgo a Escala Local y Sensible al Género: Experiencia y Aprendizajes desde Acapulco, México. Revista de Estudios Latinoamericanos sobre Reducción del Riesgo de Desastres REDER, 5(1), 13-27. http://revistareder.com/handle-0719-8477-2020-083

Rasse, A. (2019) La crisis de la vivienda: entre el derecho social y la oferta inmobiliaria. En: Hilos Tensados. Para leer el octubre chileno. USACH

Roy, A. (2005). Urban Informality: Toward an Epistemology of Planning. Journal of the American Planning Association, 71(2), 147-158.

TECHO. (2017). Encuesta de Antecedentes Previos (eap). TECHO-Chile.

TECHO, Fundación Vivienda \& CES (2021). Catastro Nacional de Campamentos 2020-2021. Informe ejecutivo. CES. https://ceschile.org/wp-content/uploads/2020/11/Informe\%2oEjecutivo_Catastro\%2o Campamentos\%202020-2021.pdf

Tironi, M., Campos, K., Acuña, V., Bonelli, C., González, M., Isola, E., Juzam, L., Kelly, S., Molina, F., Pereira, A., Rivas, R., Undurraga, B. \& Valdivieso, S. (s/f). Interruptions: imagining an analytical otherwise for disaster studies in Latin America. Disaster Prevention and Management. En preparación.

Sandoval, V. E Sarmiento, J.P. (2018). Una mirada sobre la gobernanza del riesgo y la resiliencia urbana en América Latina y El Caribe: Los asentamientos informales en la Nueva Agenda Urbana. Revista de Estudios Latinoamericanos sobre Reducción del Riesgo de Desastres REDER, 2(1), 38-52. http:// revistareder.com/handle-0719-8477-2018-011

Sandoval, V. \& Sarmiento, J.P. (2020). A Neglected Issue: Informal Settlements, Urban Development, and Disaster Risk Reduction in Latin America and the Caribbean. Disaster Prevention and Management, 29(5), 731-745. https://doi.org/10.1108/DPM-04-2020-0115

Satterthwaite, D., Archer, D., Colenbrander, S., Dodman, D., Hardoy, J., \& Patel, S. (2018). Responding to climate change in cities and in their informal settlements and economies. International Institute for Environment and Development.

Shrestha, S., \& Gaillard, J. (2013). Small-scale disasters and the recovery process. Sustainable PostDisaster Reconstruction: From Recovery to Risk Reduction, 43-54.

Solecki, W., Pelling, M. \& Garschagen, M. (2017). Transitions between risk management regimes in cities. Ecology and Society, 22(2), 38. https://doi.org/10.5751/ES-09102-220238

United Nations UN. (1997). Glossary of environment statistics, studies in methods. United Nations.

UN-Habitat. (2003). The Challenge of Slums. Earthscan.

Valdes, T., y Weinstein, M. (1993). Mujeres que sueñan: Las organizaciones de pobladoras, 1973-1989. FLACSO.

Walker, B., \& Westley, F. (2011). Perspectives on resilience to disasters across sectors and cultures. Ecology and Society, 16(2), 4. http://www.ecologyandsociety.org/vol16/iss2/art4/

Walsh, F. (2002). Bouncing forward: Resilience in the aftermath of September 11. Family Process, 41(1), 34 . 
Ward, P.M., Huerta, E.R.J., Di Virgilio, M., \& Sierra, A.C. (2016). Políticas de vivienda en ciudades latinoamericanas. Editorial Universidad del Rosario.

Weichselgartner, J., \& Kelman, I. (2015). Geographies of resilience: Challenges and opportunities of a descriptive concept. Progress in Human Geography, 39(3), 249-267.

Welsh, M. (2014). Resilience and responsibility: governing uncertainty in a complex world. The geographical journal, $180(1), 15-26$.

Wisner, B., Blaikie, P., Cannon, T., \& Davis, I. (2004). At risk: natural hazards, people's vulnerability and disasters. Psychology Press.

World Bank Group. (2020). COVID-19 and the Urban Poor Addressing those in slums. The World Bank.

Yin, R.K. (2015). Qualitative research from start to finish. Guilford Publications. 